\title{
Implementation of an Intensive Telehealth Intervention for Rural Patients with Clinic-Refractory Diabetes
}

\author{
Elizabeth A. Kobe, BS ${ }^{7}$, Allison A. Lewinski, PhD ${ }^{2,3}$, Amy S. Jeffreys, MStat', \\ Valerie A. Smith, DrPH ${ }^{2,4,5}$, Cynthia J. Coffman, PhD'2,6, Susanne M. Danus, BS ${ }^{2}$, \\ Elisabeth Sidoli, RN, BSN ${ }^{7}$, Beth D. Greck, PharmD, BCACP, CDE', \\ Leanne Horne, RN, BSN ${ }^{8}$, David R. Saxon, MD, MS $S^{9,10}$, Susan Shook, MSN, CDCES ${ }^{1}$, \\ Lina E. Aguirre, $M D, M S^{17}$, Mary G. Esquibel, Pharm D, $C D E^{11}$, \\ Clarene Evenson, RN MSN ${ }^{12}$, Christopher Elizagaray, PharmD, BCPS, CDCES ${ }^{12}$, \\ Vivian Nelson, DHA, MSN, CNS, RN-BC ${ }^{13}$, Amanda Zeek, PharmD, BCACP'3, \\ William G. Weppner, MD, MPH ${ }^{14,15}$, Stephanie Scodellaro, $R N^{15}$, \\ Cassie J. Perdew, PharmD, BCPS ${ }^{15}$, George L. Jackson, PhD, MHA $2,4,5,16$, \\ Karen Steinhauser, $P h D^{2,4}$, Hayden B. Bosworth, $P h D^{17}$, David Edelman, $\mathrm{MD}^{2,5}$, and \\ Matthew J. Crowley, MD, MHS ${ }^{2,18}$
}

\begin{abstract}
'Duke University School of Medicine, Durham, NC, USA; ${ }^{2}$ Center of Innovation to Accelerate Discovery and Practice Transformation, Durham Veterans Affairs Health Care System, Durham, NC, USA; ${ }^{3}$ School of Nursing, Duke University School of Medicine, Durham, NC, USA; ${ }^{4}$ Department of Population Health Sciences, Duke University School of Medicine, Durham, NC, USA; 5 Division of General Internal Medicine, Department of Medicine, Duke University School of Medicine, Durham, NC, USA; ${ }^{6}$ Department of Biostatistics and Bioinformatics, Duke University School of Medicine, Durham, NC, USA; ${ }^{7}$ Western North Carolina Veteran Affairs Health Care System, Asheville, NC, USA; ${ }^{8}$ VISN 19 Rocky Mountain Regional, Denver, CO, USA; 'Division of Endocrinology, Metabolism and Diabetes, University of Colorado School of Medicine, Aurora, CO, USA; ${ }^{10}$ Division of Endocrinology, Rocky Mountain Veterans Affairs Medical Center, Aurora, CO, USA; " 1 New Mexico Veteran Affairs Health Care System, University of New Mexico School of Medicine, Albuquerque, NM, USA; ${ }^{2}$ Montana Veteran Affairs Health Care System, Kalispell, MT, USA; ${ }^{13}$ Veterans Affairs Central Ohio Healthcare System, Columbus, OH, USA; ${ }^{14}$ Division of General Internal Medicine, University of Washington School of Medicine, Seattle, WA, USA; ${ }^{15}$ Boise Veteran Affairs Medical Center, Boise, ID, USA; ${ }^{16}$ Department of Family Medicine and Community Health, Duke University School of Medicine, Durham, NC, USA; ${ }^{77}$ Department of Psychiatry \& Behavioral Sciences, Duke University School of Medicine, Durham, NC, USA; ${ }^{18}$ Division of Endocrinology, Department of Medicine, Duke University School of Medicine, Durham, NC, USA.
\end{abstract}

BACKGROUND: Rural patients with type 2 diabetes (T2D) may experience poor glycemic control due to limited access to T2D specialty care and self-management support. Telehealth can facilitate delivery of comprehensive T2D care to rural patients, but implementation in clinical practice is challenging.

OBJECTIVE: To examine the implementation of Advanced Comprehensive Diabetes Care (ACDC), an evidence-based, comprehensive telehealth intervention for clinic-refractory, uncontrolled T2D. ACDC leverages existing Veterans Health Administration (VHA) Home Telehealth (HT) infrastructure, making delivery practical in rural areas.

DESIGN: Mixed-methods implementation study.

PARTICIPANTS: 230 patients with clinic-refractory, uncontrolled T2D.

INTERVENTION: ACDC bundles telemonitoring, selfmanagement support, and specialist-guided medication management, and is delivered over 6 months using existing VHA HT clinical staffing/equipment. Patients

Prior Presentations: Portions of these data were presented at the $80^{\text {th }}$ American Diabetes Association Scientific Sessions (virtual) held on June 12, 2020.

Received June 12, 2021

Accepted November 10, 2021

Published online January 3, 2022 may continue in a maintenance protocol after the initial 6-month intervention period.

MAIN MEASURES: Implementation was evaluated using the Reach, Effectiveness, Adoption, Implementation, and Maintenance (RE-AIM) framework. The primary effectiveness outcome was hemoglobin Alc (HbAlc).

KEY RESULTS: From 2017 to 2020, ACDC was delivered to 230 patients across seven geographically diverse VHA sites; on average, patients were 59 years of age, $95 \%$ male, $80 \%$ white, and 14\% Hispanic/Latinx. Patients completed an average of 10.1 of 12 scheduled encounters during the 6-month intervention period. Model-estimated mean baseline HbAlc was 9.56\% and improved to $8.14 \%$ at 6 months ( $-1.43 \%, 95 \%$ CI: $-1.64,-1.21 ; P<.001)$. Benefits persisted at $12(-1.26 \%, 95 \%$ CI: $-1.48,-$ $1.05 ; P<.001)$ and 18 months $(-1.08 \%, 95 \% \mathrm{CI}-1.35$, $-0.81 ; P<.001)$. Patients reported increased engagement in self-management and awareness of glycemic control, while clinicians and HT nurses reported a moderate workload increase. As of this submission, some sites have maintained delivery of ACDC for up to 4 years.

CONCLUSIONS: When strategically designed to leverage existing infrastructure, comprehensive telehealth interventions can be implemented successfully, even in rural areas. ACDC produced sustained improvements in glycemic control in a previously refractory population. 
KEY WORDS: diabetes mellitus, type 2; telemedicine; rural health; health services research; implementation science.

J Gen Intern Med 37(12):3080-8

DOI: $10.1007 / \mathrm{s} 11606-021-07281-8$

(c) This is a U.S. government work and not under copyright protection in the U.S.; foreign copyright protection may apply 2021

\section{BACKGROUND}

Poor control of type 2 diabetes (T2D) is the leading cause of blindness, kidney failure, and non-traumatic lower-limb amputation in the USA. ${ }^{1}$ We have defined uncontrolled T2D that remains refractory to available clinic-based services as "clinic-refractory T2D." Because T2D is more prevalent in rural areas and rural access to specialty care is often limited, ${ }^{2-5}$ rural patients may be particularly vulnerable to clinic-refractory T2D. There is thus a need for effective management strategies for clinic-refractory T2D that are amenable to rural delivery.

Telehealth, or use of telecommunications technology to facilitate remote healthcare delivery, ${ }^{6}$ is an appropriate care strategy for rural patients with clinic-refractory T2D. Studies demonstrate that comprehensive telehealth-based approaches that target factors underlying poor T2D control can lower hemoglobin A1c (HbA1c) compared with usual care. ${ }^{7-11} \mathrm{Al}-$ though the COVID-19 pandemic has increased uptake of telephone- and video-based T2D care, ${ }^{12}$ comprehensive telehealth interventions for clinic-refractory T2D have seldom been translated into clinical practice, even when proven efficacious. ${ }^{13}$ Implementation barriers for comprehensive telehealth interventions include a lack of delivery infrastructure, reimbursement challenges, inadequate integration of patientgenerated data with electronic health records (EHR), and lack of real-world telehealth implementation studies. ${ }^{13-15}$ To assure implementation of comprehensive telehealth for T2D in clinical practice, interventions must be designed in a manner that makes translation feasible. ${ }^{16}$

The Veterans Health Administration (VHA) has invested in a nationwide Home Telehealth (HT) network that provides care coordination and telemonitoring for T2D and other chronic diseases. While telemonitoring can improve control in $\mathrm{T} 2 \mathrm{D}(\sim 0.5 \% \mathrm{HbA} 1 \mathrm{c}$ reduction), many patients with clinic-refractory $\mathrm{T} 2 \mathrm{D}$ require greater HbA1c lowering. ${ }^{9}$ To this end, we developed Advanced Comprehensive Diabetes Care (ACDC), a comprehensive telehealth intervention for patients with clinic-refractory T2D. ${ }^{17,}{ }^{18}$ Critically, ACDC is delivered entirely using existing clinical HT staffing and infrastructure, making it amenable to dissemination across VHA, including in rural settings. ACDC expands traditional HT services by combining telemonitoring with module-based selfmanagement support and specialist-guided medication management. In a prior randomized trial, ACDC improved $\mathrm{HbA} 1 \mathrm{c}$ by $1.0 \%$ relative to usual care at 6 months among patients with clinic-refractory T2D (NCT01778751). ${ }^{18}$
In collaboration with the VHA Office of Rural Health $(\mathrm{ORH}), \mathrm{ACDC}$ has now been implemented at nine VHA sites serving rural populations. This manuscript describes the process of implementing and evaluating this comprehensive telehealth intervention for clinic-refractory $\mathrm{T} 2 \mathrm{D}$ in rural patients over a 4-year period.

\section{METHODS}

\section{Project Overview}

This ongoing, multi-site, single-arm evaluation project examines rural implementation of the ACDC intervention. The aims at each implementing site are to (1) adapt ACDC for local context; (2) implement HT-based delivery of ACDC; and (3) evaluate the implementation process using the Reach, Effectiveness, Adoption, Implementation, and Maintenance (REAIM) framework, which specifically seeks to improve the quality, efficiency, and impact of efforts to translate effective research into clinical practice. ${ }^{19}$

Because ACDC is an evidence-based approach with established safety and effectiveness,${ }^{18}$ its clinical delivery is covered by an Institutional Review Board (IRB) Quality Improvement exemption. As the coordinating site, the Durham Veterans Affairs Heath Care System obtained local IRB approval for implementation evaluation across sites.

In order to support ACDC's rural implementation, our team has collaborated with the VHA ORH, which has the mission to combat healthcare barriers faced by rural veterans. ${ }^{20}$ In 2020 , ORH designated ACDC a Rural Promising Practice in recognition of its potential to improve rural veterans' health and access to care. $^{21}$

\section{ACDC Intervention}

ACDC augments standard VHA HT telemonitoring with telehealth-based self-management support and specialist-guided medication management. ACDC is delivered within established scopes of HT practice, with minimal additional training required. All patients receiving ACDC are first enrolled into the local facility HT program. A HT nurse delivers ACDC's components during $\sim 30$-min synchronous telephone encounters occurring twice monthly for 6 months, for up to 12 encounters. The three ACDC components have been described in detail previously, ${ }^{18}$ and are explained briefly in Fig. 1. For the telemonitoring component, patients receive daily prompts to collect selfmonitored blood glucose (SMBG) data, which are then automatically uploaded to the EHR using HT-issued equipment (i.e., connector cable, Freestyle blood glucose meter, Medtronic HT device) and either a landline, cell phone, or internet. During phone encounters, the nurse reviews data, reconciles medications, and assesses medication adherence. For the selfmanagement support component, the nurse delivers selfmanagement education modules (developed over the course of prior trials) during most encounters. ${ }^{22,}{ }^{23}$ For the medication 


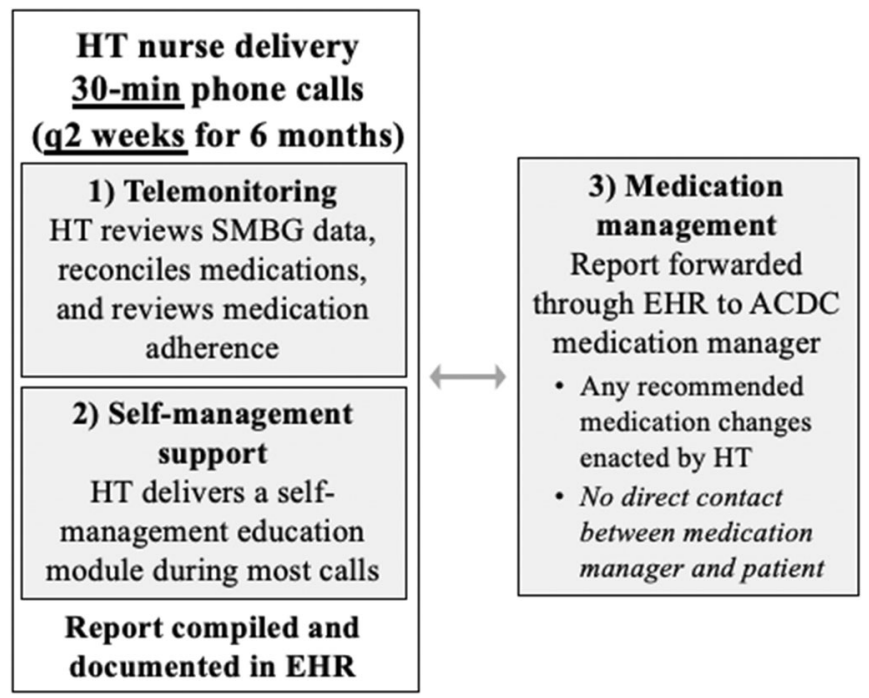

Figure 1 ACDC intervention components. Abbreviations: $\mathrm{ACDC}=$ Advanced Comprehensive Diabetes Care, $\mathrm{HT}=\mathrm{home}$ telehealth, $\mathrm{SMBG}=$ self-monitored blood glucose, $\mathrm{EHR}=$ electronic health record

management component, the nurse compiles information into a templated note that is then forwarded through the EHR to the project medication manager (typically a clinical pharmacist) for asynchronous input regarding medication changes. Suggested changes are routed back through the EHR via a templated note and enacted by the nurse. All project notes and changes are visible in the EHR.

In response to patients' desire for continued engagement beyond the initial 6-month intervention, an ACDC maintenance protocol was developed. The maintenance protocol focuses on telemonitoring and medication management ( 2 of the initial 3 core components), with every-4-week encounters continuing indefinitely. After the initial 6-month intervention, patients may continue in the ACDC maintenance protocol, continue standard HT care, or return to their previous T2D care provider, per the site teams' discretion and patient preference.

ACDC Population. The primary ACDC target population was rural patients with "clinic-refractory T2D," operationalized as HbA1c $\geq 8.5 \%$ (with goals further individualized per American Diabetes Association guidelines ${ }^{24}$ ) despite receipt of VHA care services (i.e., primary care providers and/or standard HT) during the previous year. Of note, sites could adapt these eligibility criteria based on local priorities (Appendix Table 1). Rurality was determined by linking veteran zone improvement plan (ZIP) codes to rural-urban commuting area (RUCA) codes. Patients were excluded if they used (1) continuous subcutaneous insulin infusion pumps, due to difficulty with remote medication adjustment, or (2) continuous glucose monitors (if unwilling to also transmit SMBG data per HT requirements).

\section{Site Selection}

Site selection began in 2017. Initial sites were chosen based on existing connections with the coordinating site, and later sites through broader outreach or inquiries from interested sites (Fig. 2). ORH provided initial funding to support sites in protecting time and effort from an existing HT nurse and medication manager ( 0.5 full-time equivalent (FTE) nurse and 0.25 FTE medication manager), with the aim of quickly building up delivery of ACDC. Funds were provided for up to 3 years before transitioning to site-based support for long-term delivery. Of note, two sites (Montana and Phoenix) participated without receiving ORH funds, Montana via ACDC's selection as a winner of VHA's 2018 Diffusion of Excellence (DoE) Shark Tank Competition. ${ }^{25-27}$

\section{Aim 1. Adapt ACDC for Local Context}

Because ACDC was explicitly designed to use existing VHA HT staffing, infrastructure, and technological resources, all sites had requisite HT capabilities to implement ACDC. However, to maximize suitability for implementation, the coordinating team discussed any necessary site-specific adaptations to ACDC's content and context prior to implementation. Table 1 provides a summary of ACDC core components and adaptations.

\section{Aim 2: Implement HT-Based Delivery of ACDC}

The coordinating team utilized a mentored-implementation approach to guide uptake of ACDC at new sites (Fig. 2). After engaging potential implementation sites and identifying local champion(s), an ACDC Project Toolkit was shared to guide implementation at each site; this toolkit contained all requisite materials for delivering ACDC. Once site leadership agreed to proceed with implementation, ORH funds were provided (if applicable), nurse(s) and medication manager(s) were identified by the site, and a training call was completed with the coordinating team. After delivery began, the coordinating team held a monthly all-site teleconference as well as additional monthly individual teleconferences with each new site, 


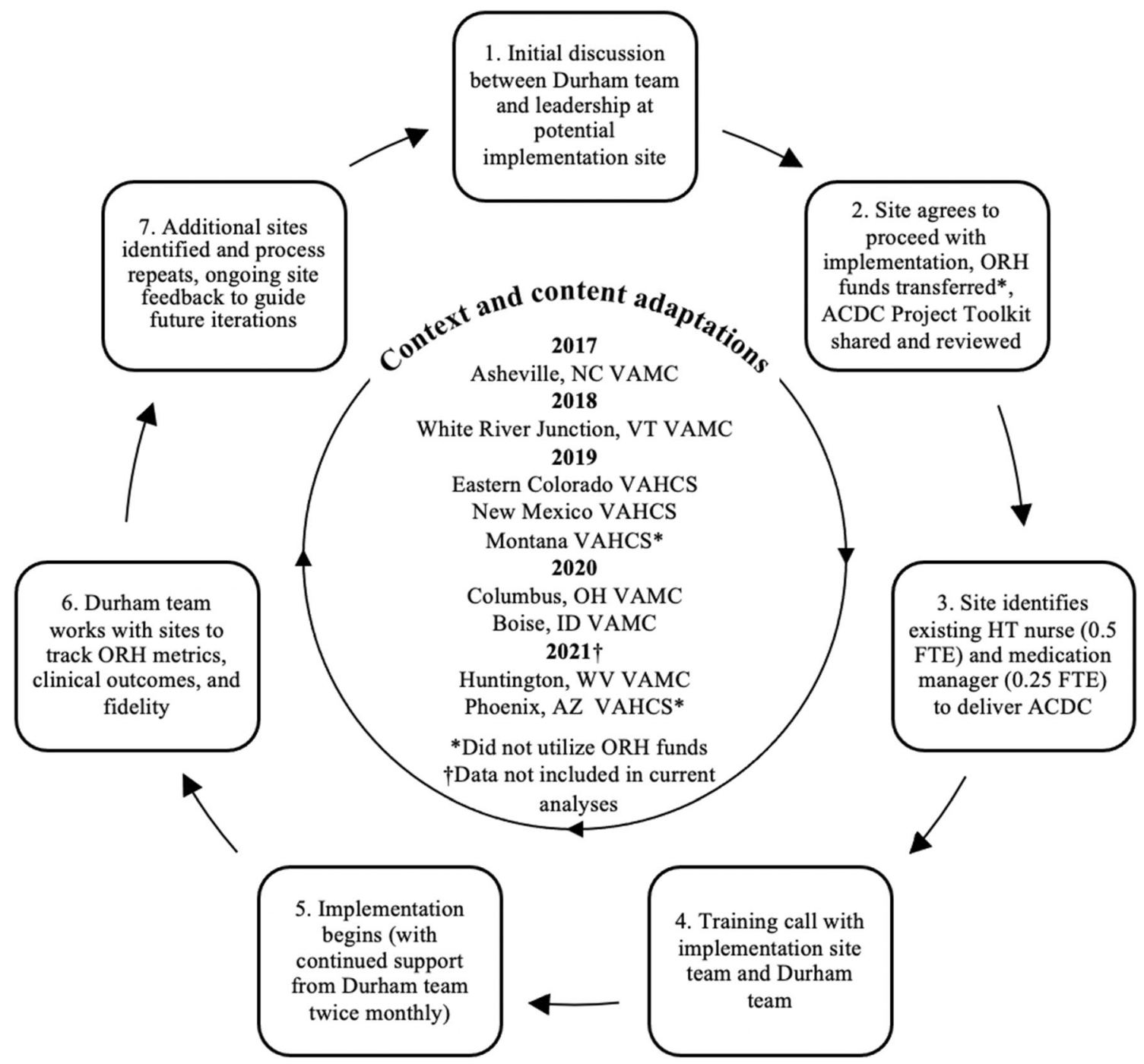

Figure 2 ACDC implementation schematic: mentored-approach, sites, and funding. Abbreviations: ORH $=$ Office of Rural Health, ACDC $=$ Advanced Comprehensive Diabetes Care, FTE $=$ full-time equivalent

in order to provide support and address problems. Of note, the all-site meetings facilitated between-site mentorship and collaboration between sites. Site feedback was used to guide improvements to ACDC and the mentored-implementation approach. The timeline from site identification to implementation was approximately 3 months.

During the COVID-19 pandemic, the coordinating team helped implementing sites navigate diversion of ACDC nurses to cover COVID patients. Delivery of ACDC continued uninterrupted at all sites during the pandemic.

\section{Aim 3. Evaluate the Implementation Process}

Our coordinating team analyzed ACDC's implementation across seven ACDC sites from 2017 to 2020 (data from 2021 implementing sites are not included, as implementation is ongoing). The implementation evaluation was guided by the RE-AIM framework. ${ }^{19}$
Outcomes. Table 2 presents each RE-AIM domain's purpose and outcome mapping to each domain. The primary outcome was HbAlc obtained from the EHR. All other quantitative metrics were tracked by individual sites using spreadsheets developed by the coordinating team.

Quantitative Analyses. For the entire cohort, all clinical HbA1c measurements were extracted from the EHR starting 1 year prior to each patient's ACDC enrollment (average 2.7 measurements/subject) through September 2020 (average 3.5 measurements/subject). Linear mixed models were fit with patient-level random intercepts and slopes for time to model HbAlc over time to estimate mean changes in HbAlc during and after the intervention period. This approach implicitly accommodates missingness when missingness is due to prior outcomes or other variables in the model. ${ }^{28}$ All data for all individuals were included, regardless of degree of intervention 
Table 1 ACDC Core Components and Adaptation(s)

\begin{tabular}{|c|c|c|c|}
\hline Domain & Relevant constructs & Core components & Adaptation(s) \\
\hline \multirow[t]{4}{*}{ Context } & Patient population & $\begin{array}{l}\text { Focus on rural patients with poorly controlled } \\
\text { diabetes }\end{array}$ & $\begin{array}{l}\text { HbA1c inclusion criteria based on site priorities and } \\
\text { needs, variability in emphasis on rural patients }\end{array}$ \\
\hline & Format & $\begin{array}{l}\text { HT nurse telephone encounters every } 2 \text { weeks for } 6 \\
\text { months }\end{array}$ & $\begin{array}{l}\text { Developed maintenance protocol in response to } \\
\text { patient feedback for those who complete the initial 6- } \\
\text { month project period }\end{array}$ \\
\hline & Delivery setting & $\begin{array}{l}\text { Delivery using only existing VHA HT infrastructure, } \\
\text { staffing, and equipment (i.e., no new hiring or needed } \\
\text { equipment) }\end{array}$ & $\mathrm{n} / \mathrm{a}$ \\
\hline & Personnel & $\begin{array}{l}\text { ACDC core staff includes (1) a HT nurse who works } \\
\text { directly with patients to deliver ACDC and (2) a } \\
\text { medication manager who works in conjunction with } \\
\text { the HT nurse to deliver the medication management } \\
\text { component }\end{array}$ & $\begin{array}{l}\text { 1-5 HT nurses used at each site; Asheville, White } \\
\text { River Junction, New Mexico, Montana, Columbus, } \\
\text { and Boise used a clinical pharmacist (PharmD) } \\
\text { medication manager, while Eastern Colorado used an } \\
\text { endocrinologist (MD) }\end{array}$ \\
\hline \multirow[t]{5}{*}{ Content } & Intervention procedures & $\begin{array}{l}\text { ACDC intervention core components include }(1) \\
\text { telemonitoring, (2) self-management support (12 } \\
\text { intervention modules), and (3) medication manage- } \\
\text { ment (Fig. 1) }\end{array}$ & $\begin{array}{l}\text { In response to HT nurse feedback, refinements were } \\
\text { made to self-management support modules, and one } \\
\text { new module (sick day care) was added. }\end{array}$ \\
\hline & Intervention materials & $\begin{array}{l}\text { Patients perform self-monitoring of blood glucose } \\
\text { data (SMBG) using standard HT-issued equipment }\end{array}$ & $\mathrm{n} / \mathrm{a}$ \\
\hline & & $\begin{array}{l}\text { HT nurse documents a summary note in CPRS for } \\
\text { each encounter to facilitate communication with } \\
\text { medication manager }\end{array}$ & $\begin{array}{l}\text { Minor site-specific updates to project note templates } \\
\text { were created in CPRS using each site's OIT team to } \\
\text { suit local HT nurse and medication manager prefer- } \\
\text { ences }\end{array}$ \\
\hline & $\begin{array}{l}\text { Intervention } \\
\text { implementation and }\end{array}$ & $\begin{array}{l}\text { Standardized, mentored-implementation approach } \\
\text { (Fig. 2) }\end{array}$ & $\begin{array}{l}\text { Site feedback guided subsequent iterations (e.g., } \\
\text { development of maintenance protocol) }\end{array}$ \\
\hline & ongoing support & $\begin{array}{l}\text { Identification of local site champion and continued } \\
\text { engagement with mentoring team in Durham }\end{array}$ & $\begin{array}{l}\text { Added monthly, all-site video conferences to foster } \\
\text { mentorship collaboration across sites in } 2020\end{array}$ \\
\hline
\end{tabular}

Abbreviations: VHA Veterans Health Administration, HT Home Telehealth, ACDC Advanced Comprehensive Diabetes Care, CPRS Computerized Patient Record System

*See Appendix Table 1 for complete patient inclusion criteria and identification methods per study site

engagement. The primary model included linear terms for number of days from enrollment to capture the 1-year period prior to enrollment, and interaction terms for number of days from enrollment interacting with indicators for the intervention period, and post-intervention period to allow trends in $\mathrm{HbAlc}$ to differ in these distinct periods. A second model was fit to estimate the impact of the maintenance program compared to return to PCP care for patients enrolled early enough to have sufficient follow-up. For other RE-AIM domains, we descriptively examined outcome variables listed in Table 2.
Qualitative Analyses. Semi-structured interviews were conducted to obtain actionable data on ACDC's feasibility and acceptability. Each year, 5-20 veterans (total $n=38$ ) were invited to participate in interviews after completion of the 6-month intervention, and 3-5 VHA staff and administrators (total $n=15$ ) after 1 year of site participation; each year, interviews were stopped once information saturation was achieved. $^{29}$ These 15-20-min interviews were conducted with a diverse sample of individuals (based on baseline $\mathrm{HbA}$ c and race) and recorded by a trained research assistant via telephone. Interview questions focused on overall experience

Table 2 RE-AIM Framework, Purpose, and Outcome Variables

\begin{tabular}{|c|c|c|}
\hline $\begin{array}{l}\text { RE-AIM } \\
\text { dimension }\end{array}$ & Purpose & Outcome variable(s) \\
\hline Reach & Evaluate ability to engage the target rural population & $\begin{array}{l}\text { - Inclusion criteria at each site } \\
\text { - Proportion of rural/highly rural as determined by RUCA }\end{array}$ \\
\hline Effectiveness & $\begin{array}{l}\text { Determine objective impact of the intervention on study } \\
\text { outcomes }\end{array}$ & $\begin{array}{l}\text { - Pre/post HbAlc change within implementation cohort (primary } \\
\text { outcome) } \\
\text { - Difference in HbA1c change between patients receiving and not } \\
\text { receiving maintenance protocol after initial 6-month intervention }\end{array}$ \\
\hline Adoption & $\begin{array}{l}\text { Assessment of barriers and facilitators to intervention } \\
\text { uptake and utilization by patients and individual sites }\end{array}$ & $\begin{array}{l}\text { - Qualitative patient and provider interviews of barriers/facilitators to } \\
\text { intervention uptake or utilization }\end{array}$ \\
\hline Implementation & $\begin{array}{l}\text { Determine extent to which intervention is delivered as } \\
\text { intended }\end{array}$ & $\begin{array}{l}\text { - Number of completed intervention modules } \\
\text { - Average duration of encounters }\end{array}$ \\
\hline Maintenance & $\begin{array}{l}\text { Assessment of patient and site readiness to maintain the } \\
\text { intervention }\end{array}$ & $\begin{array}{l}\text { - Number of years sites have been delivering ACDC } \\
\text { - Number of patients entering the maintenance protocol } \\
\text { - Qualitative patient and provider interviews of barriers/facilitators to } \\
\text { intervention maintenance }\end{array}$ \\
\hline
\end{tabular}


engaging with or delivering ACDC, perceptions of core components, integration into normal workflow, and/or challenges with delivery.

Data were analyzed using thematic analysis and the matrix method. ${ }^{30-32}$ A coding team consisting of three individuals with qualitative expertise developed a priori codes based on core components of ACDC, ${ }^{17}$ and feasibility and acceptability of engaging in, or delivering, ACDC. The coding team independently read and coded each transcript, resolved coding differences through discussion, and then grouped similar codes into larger themes. Themes and findings were discussed with the larger team during analysis.

\section{RESULTS}

\section{Population Characteristics}

From 2017 to 2020, ACDC engaged 230 patients across seven sites: Asheville, White River Junction, Eastern Colorado, New Mexico, Montana, Columbus, and Boise. The implementation cohort was predominantly male $(94.8 \%)$ and majority white $(80.0 \%)$, with a mean age of 59 years (Table 3 ). The population had a model-estimated mean baseline $\mathrm{HbA1c}$ of $9.56 \%$.

\section{RE-AIM Evaluation}

Reach. Specific inclusion criteria and patient recruitment differed by site (Appendix Table 1). All sites met the suggested criteria for clinic-refractory T2D (see "ACDC

Table 3 ACDC2 Implementation Cohort Data

\begin{tabular}{ll}
\hline \hline Baseline characteristics & $\begin{array}{l}\mathbf{2 0 1 7 - 2 0 2 0} \text { cohort } \\
(\boldsymbol{n}=\mathbf{2 3 0 )}\end{array}$ \\
\hline $\begin{array}{l}\text { Demographic characteristics } \\
\text { Age (years), mean (SD) }\end{array}$ & $59.4(1.1)$ \\
Male, $n(\%)$ & $218(94.8)$ \\
Race, $n(\%)$ & $184(80.0)$ \\
Caucasian & $20(8.7)$ \\
African American & $7(3.0)$ \\
American Indian/Alaska Native & $2(0.9)$ \\
Hawaiian/Pacific Islander & $17(7.4)$ \\
Declined/missing & $33(14.4)$ \\
Hispanic/Latino ethnicity, $n(\%)$ & $13(5.7)$ \\
Rurality (by RUCA), $n(\%)$ & $132(57.4)$ \\
Highly rural & $84(36.5)$ \\
Rural & $1(0.4)$ \\
Urban & \\
Missing & $9.56(1.5)$ \\
Clinical characteristics & \\
Baseline HbAlc, mean (SD) & \\
Site recruitment characteristics & 56 \\
Total patients engaged, $n$ & 13 \\
Asheville & 58 \\
White River Junction & 42 \\
Eastern Colorado & 21 \\
New Mexico & 24 \\
Montana & 16 \\
Columbus & \\
Boise &
\end{tabular}

Abbreviation: RUCA rural-urban commuting area
Population"), and $63.0 \%$ of the cohort classified as rural/ highly rural per RUCA codes.

Effectiveness. At 6 months, mean $\mathrm{HbA1c}$ improved by $1.43 \%$ ( $95 \%$ confidence interval $(\mathrm{CI}),-1.64$ to $-1.21 ; p<0.001$ ) across the implementation cohort (Table 4). This difference was largely maintained at 12 months (estimated mean difference from baseline, $-1.26,95 \%(\mathrm{CI}),-1.48$ to $-1.05 ; p<$ 0.001 ) and 18 months (estimated mean difference from baseline, $-1.08,95 \%(\mathrm{CI}),-1.35$ to $-0.81 ; p<0.001)$ across the cohort. Differences in HbA1c change between patients entering the maintenance protocol and those returning to usual care after the 6-month intervention were not statistically significant (between-group estimated mean difference at 12 months $0.21 \%, 95 \%$ (CI), -0.43 to 0.005 ; between-group estimated mean difference at 18 months $-0.43 \%, 95 \%$ (CI), -0.86 to 0.01). Of note, at the time of these analyses, the Columbus and Boise sites had not accrued sufficient time for patients to transition to the maintenance protocol. While hypoglycemia rates were not tracked uniformly across sites, there were no reports of untoward hypoglycemia, consistent with the initial ACDC trial. ${ }^{18}$

Adoption. Interviews to date indicate the following: (1) patients benefitted from the repeated and frequent contact from the ACDC nurse, who provided personal attention and support for T2D self-management; (2) synchronous interactions with the ACDC nurse allowed patients to question and receive realtime feedback, enhancing their engagement; (3) staff enjoyed the team dynamic ACDC fostered, particularly supporting collaboration between the nurse and medication manager; (4) patients expressed improved awareness of their T2D control as well as a sense of accountability for their self-management; and (5) staff acknowledged that ACDC did increase their workload.

Implementation. Of individuals receiving the first module in 2020 , an average of 8.9-11.3 of 12 scheduled modules were completed per patient at each site (combined average 10.1 (SD $=3.3)$ ) with an average call length of $24.3 \mathrm{~min}$. Interview findings describe how each site adapted delivery of ACDC to improve "fit" and local contextual relevance. Sites differed modestly on their approaches to selecting HT nurses for

Table 4 Predicted Mean HbA1c and Estimated Differences in HbA1c (95\% CI) from Baseline

\begin{tabular}{lll}
\hline \hline $\begin{array}{l}\text { Time } \\
\text { point }\end{array}$ & $\begin{array}{l}\text { Predicted mean } \\
\text { HbA1c }(95 \% \text { CI) }\end{array}$ & $\begin{array}{l}\text { Predicted mean HbA1c } \\
\text { difference from baseline }(95 \% \\
\text { CI) }\end{array}$ \\
\hline $\begin{array}{lll}\text { Baseline } \\
6 \text { months }\end{array}$ & $9.56(9.37,9.76)$ & - \\
$\begin{array}{l}12 \\
\text { months }\end{array}$ & $8.30(8.11,8.5 .50)$ & $-1.43(-1.64,-1.21)$ \\
$\begin{array}{l}18 \\
\text { months }\end{array}$ & $8.48(8.24,8.73)$ & $-1.08(-1.35,-0.81)$ \\
\hline
\end{tabular}


participation (e.g., single nurse versus multiple), defining the population of eligible veterans, specific approaches to HT nurse/medication manager interactions, and activities during long-term maintenance of ACDC (Appendix Table 1).

Maintenance. With the exception of one site, which experienced HT staffing turnover during 2018 leading to suspension of ACDC, all other sites continue to maintain ACDC delivery. Asheville is currently in its fourth year delivering ACDC, and three sites (Asheville, Eastern Colorado, New Mexico) no longer use ORH financial support. To justify the financial feasibility of transitioning from ORH funding to more sustainable site-based funding, program staff developed a detailed ACDC Business Plan outlining program costs, savings, and revenues. Of the 129 patients across all sites who completed the 6-month intervention by the end of 2020, 39 transitioned to the maintenance protocol and 90 returned to standard care alone.

\section{DISCUSSION}

While comprehensive telehealth interventions for T2D have proven efficacious in research settings, such interventions are rarely implemented in clinical practice. An average of 17 years is typically required for integration of effective research interventions into clinical care, and only $14 \%$ successfully make this transition. ${ }^{33}$ Narrowing this implementation gap demands strategically designed interventions like ACDC. ${ }^{13}$ ACDC lowered $\mathrm{HbA} 1 \mathrm{c}$ by an estimated $1.43 \%$ over 6 months, an improvement that was largely maintained at 12 and 18 months. ${ }^{9-11}$ This improvement should translate to meaningful reductions in T2D sequelae and costs over time, ${ }^{34-36}$ a $1 \%$ improvement in $\mathrm{HbA} 1 \mathrm{c}$ has been associated with a $21 \%$ drop in overall T2D complications, $21 \%$ reduction in T2D-related death, and a $37 \%$ reduction in microvascular complications. ${ }^{36}$ ACDC's effectiveness is particularly noteworthy when considering that its target population had already proven refractory to clinic-based T2D care.

This project highlights several critical elements for successful implementation. ACDC's three components (telemonitoring, self-management, and medication management) were designed to target key factors underlying poor glycemic control, including unreliable or unavailable blood glucose data, self-management non-adherence, and complex medication regimens, respectively. ${ }^{11,37,38}$ Data from qualitative interviews indicated these components were acceptable and successful at the patient, provider, and organizational levels. Critically, ACDC required only existing clinical staffing and infrastructure for implementation. HT services are well established across VHA nationwide, including rural areas, and so provide a widely accessible pathway for remote delivery of intensive, specialized T2D care. ${ }^{39}$ Ultimately, ACDC's early focus on feasible delivery promoted smooth implementation and yielded unique potential for scaling within VHA. ${ }^{16}$

Because ACDC leverages strong operational partnerships, early assessment of site readiness and uptake barriers, tailored delivery to meet local needs, cultivation of relationships with local champions, and collaboration between implementation sites, the intervention has high potential for sustainment. We have encouraged site feedback to guide future implementation, which has led to development of the maintenance protocol and improved ease of engagement, recruitment, and implementation at new sites. Despite the COVID-19 pandemic, ACDC's implementation has continued unimpeded due to its telehealth-based design and use of existing infrastructure. ACDC's resilience during the pandemic highlights its ability to overcome limitations of traditional office-based care.

Throughout this implementation process, we have learned valuable lessons. First, HT staffing turnover led to suspension of the ACDC program at an early site, leading to a more thorough exploration of the stability of candidate sites' HT programs going forward. Second, identification of strong leadership at implementation sites and collaboration with key stakeholders (e.g., ORH and DoE) have contributed greatly to ACDC's success and sustainment. Third, staff and administration feedback described moderately increased provider workload; while our target population's clinic-refractory T2D justifies intensive intervention, early establishment of staff expectations assured that ACDC was accepted and highly valued by both patients and staff. Lastly, differing site processes contributed to occasional challenges with implementation data collection; as such, expectations regarding data collection processes are now clarified in advance of intervention delivery.

\section{Limitations}

This was a single-arm project focusing on a primarily rural, male, veteran population with clinic-refractory T2D, so findings should be interpreted accordingly. Additional work should explore ACDC's acceptability to populations underrepresented in our cohort (e.g., younger, female, and gender minority patients). The intervention leverages existing VHA HT services and equipment, which limits generalizability beyond VHA. However, our processes of strategic intervention design, site and patient engagement, mentored implementation, and evaluation likely have broad applicability. Use of HT also limited inclusion of patients currently using continuous glucose monitors or insulin pumps, but as HT continues to explore incorporation of these newer technologies, ACDC will likewise do so. While our maintenance protocol analysis was limited by a small sample size, we will address this limitation as ACDC continues to expand.

\section{CONCLUSIONS}

ACDC addresses current gaps in rural T2D care by leveraging ubiquitous VHA telehealth resources for practical, effective 
management of clinic-refractory T2D. ACDC's impact is particularly impressive given its target population's established resistance to clinic-based T2D care. With ACDC, rural patients can now access comprehensive T2D care that has often been unavailable. With the rapid increase in telehealth usage in response to COVID-19, ${ }^{40-42}$ comprehensive interventions like ACDC are well positioned to have an enduring impact on clinical practice.

Supplementary Information The online version contains supplementary material available at https://doi.org/10.1007/s11606-02107281-8.

\section{Contributors: N/A}

Corresponding Author: Matthew J. Crowley, MD, MHS; Division of Endocrinology, Department of Medicine, Duke University School of Medicine, Durham, NC, USA (e-mail: matthew.crowley@duke.edu).

Funding - Veterans Affairs Office of Rural Health (ORH Award Number 13366).

- Veterans Affairs Diffusion of Excellence Program.

- Veterans Affairs Quality Enhancement Research Initiative (QUERI) (QUE 12-458, 20-012).

- The research reported in this publication was supported by Durham Center of Innovation to Accelerate Discovery and Practice Transformation grant \#CIN 13-410.

- EAK is supported by the National Center for Advancing Translational Sciences of the National Institutes of Health (NIH, TL1 TROO2555).

- Dr. Lewinski is supported by VA HSR\&D Nursing Research Initiative grant (18-234).

- Dr. Steinhauser is supported by NIH (1U54-MD012530-01 and R01CA229425-01A1) and the Patient-Centered Outcomes Research Institute (R-1609-36381).

- Dr. Bosworth is supported by VA HSRD Senior Career Scientist award (VA HSR\&D 08-027).

- Dr. Crowley is supported by VA HSR\&D (IIR 16-213), VA QUUER (QUE 20-012), the VA Office of Rural Health (ORH Award Number 13366), and NIH (1RO1NRO19594-01).

The content is solely the responsibility of the authors and does not reflect the position or policy of Duke University, the U.S. Department of Veterans Affairs, or the U.S. government.

\section{Declarations:}

Conflict of Interest: Dr. Bosworth reports research grants from the PhRMA Foundation, Proteus Digital Health, Otsuka, Novo Nordisk, Sanofi, Improved Patient Outcomes, Boehinger Ingelheim, NIH, and VA as well as consulting from Sanofi, Novartis, Otsuka, Abbott, Xcenda, Preventric Diagnostics, Boehringer Ingelheim, and the Medicines Company. None of this work relates to the current study. Dr. Lewinski reports receiving funds from PhRMA Foundation and Otsuka; none of this work relates to the current study. The remaining authors have no competing interests to declare.

\section{REFERENCES}

1. Engelgau MM, Geiss LS, Saaddine JB, et al. The evolving diabetes burden in the United States. Ann Intern Med. 2004;140(11):945-950.

2. Mainous AG, 3rd, King DE, Garr DR, Pearson WS. Race, rural residence, and control of diabetes and hypertension. Ann Fam Med. 2004;2(6):563568.

3. O'Connor A, Wellenius G. Rural-urban disparities in the prevalence of diabetes and coronary heart disease. Public Health. 2012;126(10):813820 .

4. Strauss K, MacLean C, Troy A, Littenberg B. Driving distance as a barrier to glycemic control in diabetes. J Gen Intern Med. 2006;21(4):378-380.
5. Zgibor JC, Gieraltowski LB, Talbott EO, Fabio A, Sharma RK, Hassan K. The association between driving distance and glycemic control in rural areas. J Diabetes Sci Technol. 2011;5(3):494-500.

6. NEJM Catalyst. What Is Telehealth? https://catalyst.nejm.org/doi/full/ 10.1056/CAT.18.0268. Published 2018. Accessed December 6, 2021

7. Aburizik A, Dindo L, Kaboli P, Charlton M, Dawn K, Turvey C. A pilot randomized controlled trial of a depression and disease management program delivered by phone. J Affect Disord. 2013;151(2):769-774.

8. Faruque LI, Wiebe N, Ehteshami-Afshar A, et al. Effect of telemedicine on glycated hemoglobin in diabetes: a systematic review and meta-analysis of randomized trials. CMAJ. 2017;189(9):E341-E364.

9. Medical Advisory S. Home telemonitoring for type 2 diabetes: an evidence-based analysis. Ont Health Technol Assess Ser. 2009;9(24):1-38.

10. Medical Advisory S. Behavioural interventions for type 2 diabetes: an evidence-based analysis. Ont Health Technol Assess Ser. 2009;9(21): 1-45.

11. Pimouguet C, Le Goff M, Thiebaut R, Dartigues JF, Helmer C. Effectiveness of disease-management programs for improving diabetes care: a meta-analysis. CMAJ. 2011;183(2):E115-127.

12. Patel SY, Mehrotra A, Huskamp HA, Uscher-Pines L, Ganguli I, Barnett ML. Trends in Outpatient Care Delivery and Telemedicine During the COVID-19 Pandemic in the US. JAMA Intern Med. 2021;181(3):388-391.

13. Glasgow RE, Lichtenstein E, Marcus AC. Why don't we see more translation of health promotion research to practice? Rethinking the efficacy-to-effectiveness transition. Am J Public Health. 2003;93(8):12611267.

14. Green LW. From research to "best practices" in other settings and populations. Am J Health Behav. 2001;25(3):165-178.

15. Orlandi MA. Promoting health and preventing disease in health care settings: an analysis of barriers. Prev Med. 1987;16(1):119-130.

16. Milat AJ, King L, Bauman AE, Redman S. The concept of scalability: increasing the scale and potential adoption of health promotion interventions into policy and practice. Health Promot Int 2013;28(3):285-298.

17. Andrews SM, Sperber NR, Gierisch JM, et al. Patient perceptions of a comprehensive telemedicine intervention to address persistent poorly controlled diabetes. Patient Prefer Adherence. 2017;11:469-478.

18. Crowley MJ, Edelman D, McAndrew AT, et al. Practical Telemedicine for Veterans with Persistently Poor Diabetes Control: A Randomized Pilot Trial. Telemed J E Health. 2016;22(5):376-384.

19. Glasgow RE, Vogt TM, Boles SM. Evaluating the public health impact of health promotion interventions: the RE-AIM framework. Am J Public Health. 1999;89(9):1322-1327.

20. U.S. Department of Veterans Affairs. Office of Rural Health. https://www. ruralhealth.va.gov. Published 2020. Accessed Jan 9, 2020.

21. U.S. Department of Veterans Affairs. Rural Promising Practices - Office of Rural Health. https://www.ruralhealth.va.gov/providers/promising practices.asp. Published 2019. Accessed Jan 9, 2021.

22. Crowley MJ, Bosworth HB, Coffman CJ, et al. Tailored Case Management for Diabetes and Hypertension (TEACH-DM) in a community population: study design and baseline sample characteristics. Contemp Clin Trials. 2013:36(1):298-306.

23. Crowley MJ, Powers BJ, Olsen MK, et al. The Cholesterol, Hypertension, And Glucose Education (CHANGE) study: results from a randomized controlled trial in African Americans with diabetes. Am Heart J. 2013;166(1):179-186.

24. American Diabetes A. 6. Glycemic Targets: Standards of Medical Care in Diabetes-2020. Diabetes Care. 2020;43(Suppl 1):S66-S76.

25. Jackson GL, Cutrona SL, White BS, et al. Merging Implementation Practice and Science to Scale Up Promising Practices: The Veterans Health Administration (VHA) Diffusion of Excellence (DoE) Program. Jt Comm J Qual Patient Saf. 2021;47(4):217-227.

26. Nevedal AL, Reardon CM, Jackson GL, et al. Implementation and sustainment of diverse practices in a large integrated health system: a mixed methods study. Implement Sci Commun. 2020;1:61.

27. Vega R, Jackson GL, Henderson B, et al. Diffusion of Excellence: Accelerating the Spread of Clinical Innovation and Best Practices across the Nation's Largest Health System. Perm J. 2019;23.

28. Little RJA, Rubin DB. Statistical analysis with missing data. 2nd ed. Hoboken, N.J.: Wiley; 2002.

29. Malterud K, Siersma VD, Guassora AD. Sample Size in Qualitative Interview Studies: Guided by Information Power. Qual Health Res. 2016;26(13):1753-1760.

30. Gale NK, Heath G, Cameron E, Rashid S, Redwood S. Using the framework method for the analysis of qualitative data in multidisciplinary health research. BMC Med Res Methodol. 2013;13:117. 
31. Thomas J, Harden A. Methods for the thematic synthesis of qualitative research in systematic reviews. BMC Med Res Methodol. 2008;8:45.

32. Averill JB. Matrix analysis as a complementary analytic strategy in qualitative inquiry. Qual Health Res. 2002;12(6):855-866.

33. Balas EA. From appropriate care to evidence-based medicine. Pediatr Ann. 1998;27(9):581-584.

34. Gilmer TP, O'Connor PJ, Manning WG, Rush WA. The cost to health plans of poor glycemic control. Diabetes Care. 1997;20(12):1847-1853.

35. McBrien KA, Manns BJ, Chui B, et al. Health care costs in people with diabetes and their association with glycemic control and kidney function. Diabetes Care. 2013;36(5): 1172-1180.

36. Stratton IM, Adler AI, Neil HA, et al. Association of glycaemia with macrovascular and microvascular complications of type 2 diabetes (UKPDS 35): prospective observational study. BMJ. 2000;321(7258):405-412.

37. Crowley MJ, Holleman R, Klamerus ML, Bosworth HB, Edelman D, Heisler M. Factors associated with persistent poorly controlled diabetes mellitus: clues to improving management in patients with resistant poor control. Chronic Illn. 2014;10(4):291-302.
38. Davidson J. Strategies for improving glycemic control: effective use of glucose monitoring. Am J Med. 2005; 118(Suppl 9A):27S-32S.

39. Hale NL, Bennett KJ, Probst JC. Diabetes care and outcomes: disparities across rural America. J Community Health. 2010;35(4):365-374.

40. Centers for Medicare \& Medicaid Services. Medicare Telemedicine Health Care Provider Fact Sheet. https://www.cms.gov/newsroom/fact-sheets/ medicare-telemedicine-health-care-provider-fact-sheet. Published 2020. Accessed December 6, 2021.

41. United States Department of Health \& Human Services. Notification of Enforcement Discretion for Telehealth Remote Communications During the COVID-19 Nationwide Public Health Emergency.

42. Mann DM, Chen J, Chunara R, Testa PA, Nov O. COVID-19 transforms health care through telemedicine: Evidence from the field. $J$ Am Med Inform Assoc. 2020;27(7):1132-1135.

Publisher's Note: Springer Nature remains neutral with regard to jurisdictional claims in published maps and institutional affiliations. 